\title{
Discussion on Inspiration Theory in Chinese Classical Aesthetics
}

\author{
Ying Chen \\ College of Literature, Dalian University, Liaoning, China \\ Email: clariey1102@163.com
}

How to cite this paper: Chen, Y. (2017). Discussion on Inspiration Theory in Chinese Classical Aesthetics. Advances in Literary Study, 5, 94-104.

https://doi.org/10.4236/als.2017.54008

Received: September 26, 2017

Accepted: October 22, 2017

Published: October 25, 2017

Copyright $\odot 2017$ by author and Scientific Research Publishing Inc. This work is licensed under the Creative Commons Attribution International License (CC BY 4.0).

http://creativecommons.org/licenses/by/4.0/

\begin{abstract}
The Chinese classical aesthetics starts from the simple theory of interaction between the heart and object, and has made smart and rich interpretations on inspiration thinking in art creations, and is full of the dialectical thinking flame of "unity" and "disunity": both emphasizing unexpected "cherishing by chance" and emphasizing voluminous "knowledge accumulation and treasure storage" in the potential gestation; as for the approach of acquiring inspiration, both highlighting the perceptual "tacit understanding", and highlighting the soul and object correlated "soul comprehension"; as for the stimulation and trapping of inspiration, both showing appreciation to the digression "reflection" of "ingenious nature, without fabrication", and highlighting the timely materialization of "chasing and catching in a hurry".
\end{abstract}

\section{Keywords}

Inspiration, Enlightenment, Intuition

\section{Introduction}

Inspiration is a remarkable topic in the field of aesthetics. Through the ages, aestheticians put forward many insights, accumulating plentiful research achievements. However, because of the differences between Chinese and Western culture, western aesthetics pays attention on logic, understanding and reason, profound and comprehensive. Different from it, Chinese aesthetics advocates more about nature, feeling, intuition and experiencing. Thus, the aspiration of Chinese aesthetics is usually presented in a way of empirical experience and narration. Though Chinese aesthetics lacks of the clear and insightful description in western aesthetics, it has its own characteristics of rich implications and vitality. Its attentions on the individual aesthetic experiences, sentiment of 
life in the universe and appreciation for natural charm clearly focus on the clarity in the depth of human's heart, and are full of infinite creative thinking. At the same time, they wander from unity and disunity with the dialectical thinking flame. The thesis reflects Chinese classical aesthetics inspiration theory on the basis of western aesthetics "the other mirror". It explores inspiration's potential gestation, accidental generation, the approach of acquiring and trapping inspiration in the view of psychological aesthetics. It is a simple attempting to reveal the mystery of inspiration thinking and get through artistic creation concepts of both China and the West in all the times.

\section{The Potential Gestation of Inspiration: Unexpected Cherishing by Chance and Voluminous "Knowledge Accumulation and Treasure Storage"}

Though Chinese classical aesthetics does not directly put forward the concept of "inspiration", it has a lot of theoretical discourse about the inspiration thinking, for example, "Xing”, "Gan Xing” or "Xing Hui" are prominently emphasized as the synonyms of inspiration. As Xie Zhen's Si Ming Poetry wrote: for poetry, joys and sorrows are all from Xing, if there is no Xing, all words will be in vain. In Lu Ji's $W e n ~ F u$, there is a sentence:"the creation of state is not controlled by himself but the natural mind and he can't decide whether the inspiration should go or not". Ancient Chinese often emphasized a sudden and unpredictable inspiration which is not controlled by mind. As Guan Xiu said in Yan Shi, you can hardly find Xing, but sometimes it let itself come out. Some writers even don't believe or do not recognize that the masterpiece which is written out by his or her inspiration is not his or her ideas but the god's work or help. According to Zhong Rong's Xie Huilianin of Shi Pin, in which he quoted The Xie's families Words, in which Xie Lingyun told that "the spring grass grow on the side of pond" was not written by himself but the god, and as Lu You's Article said, article is generated from nature and it just be written by chance ...

Just as Chinese aestheticians, western aestheticians also have a theory which is created through a special power from inspiration, as Claudel said, poem is just for the soul, not heart. I am here but I am not stubborn. When the writing brush is worn out, you will see the truth which is as same as the Dao's viewpoint which is "when you feel lonely you will have an emotion, then get a realm" (Qian, 1984). They are in the same place between poem and mystery, and poem is original from realm not heart, French priest Henri Bremond said," Just as what religion can communicate directly with God and convey God's words to be a prophet. when they are able to understand unknowable things, see the invisible things and insight the sightless things, the insight and the mystery of poems are also mysterious, profound and insightful (Qian, 1984). Inspiration which is viewed as God's will possessed by God is colored with a kind of mysterious color by these acquaintances is the secret and mysterious poetry simultaneously.

On the surface, in the view of Western and Chinese classical aesthetics the in- 
spiration is deified. However, in fact, they are different from each other. Poetry god is regarded as the origin of inspiration in Western classical aesthetics and it is the poetry god who possesses the writer and makes the artist getting into an obsessed creation state that a writer absolutely lost himself. As Plato thought, whether a great poem can create a poet or not, the key point is that if the writer can get the inspiration which is transported by poetry god, the inspiration theory of "as God's help" is obviously covered with a coat which is tailored by the idealism and mystical experience of religion. Compared with Western classical aesthetics, although Chinese classical aesthetics regards the coming inspiration as "poetry god's help", it pays more attention on the hidden inevitability behind contingency of inspiration, namely "Cherishing by chance". Chinese traditional aesthetics puts more attention on the efforts which are produced by subject itself in usual life and also keeps a watchful eye on the amassing experience and has an indomitable urge for thought object.

In the view of Chinese classical aestheticians, inspiration comes from the creation subject's own artistic conditions: knowledge accumulation, artistic experience and life practice are the basic conditions of gestating inspiration; long-term mental effort and persistent pursuit of artistic objects are the dynamic conditions; energy, enthusiasm and vitality are the physiological conditions; the subject's relax and clear mental state are the psychological conditions of stimulating inspiration (Zheng \& Li, 1987).

Second, long-term mental effort and persistent pursuit of artistic objects are the dynamic conditions to initiate inspiration. Chinese ancients realized that erratic inspiration especially showed appreciation for the people who has a painstaking effort with the spirit of "For you I am pining myself away without regret". It is a hard work of accumulation and recondite gestation in daily lives that enlighten the creative subject in a sudden and urge the inspiration to be burst in a twinkling. Just as Yan Yu who said in his Cang Lang Shi Hua, "inspiration brewed in heart, it will be unaffectedly understood as time passes"; In Zhao Mei Zhan Yan, Fang Dongshu pointed that "when you persist in thinking for a long time, you will make your head be full of thoughts and then have a wonderful inspiration." Inspiration is always experiencing a pop-up process when art is brewed from quantitative change to qualitative change. The more a writer pursues the thought subject, the more information the writer will accumulate in his head. Thus the mental field will have a qualitative leap. Therefore, Guan Zi considered that thinking, thinking, rethinking and then ghosts and gods would attach on your mind, but it is not the ghost or god's effort, but the vital essence which is the most important thing for the thinker. The creative subject's desire and persistent efforts are the dynamic foundation of generating inspiration which speed up the acquisition of inspiration. So the ancients always emphasize that without the hardship that you have to wear out your iron shoes, there is no delight of inspiration that you get it without effort.

Next, inspiration requires good physiological and psychological conditions. 
Liu Xie had written in his Wen Xin Diao Long. Following the development of emotional state, natural and harmonious, reconciling the train of thoughts and smoothen emotions; if one studies excessively, he will feel tired and exhausted, this is the general principle of temper. Only brimming with energy and spirit, mind and emotions will be harmonious and healthy, and thoughts will be creative and fluent; otherwise, people will be tired in spirit and energy and then have no idea and gain nothing. At the same time, Liu Xie also thought that writer's quiet psychological condition is also important: polishing the thinking lies in the visional peace, purifying one's soul and body and washing one's mind in a clean and pure word. Only in a free and relax condition can the mood focus on the object, and exclude the interference of the utilitarian heart and external things to enter a aesthetic mental state, free and divine, bringing the flow of ideas and having a ultimate state of inspiration. Therefore, in order to provide the best physical qualifications and psychological space, creative subject should have a free and relax mentality to maintain essence and energy.

\section{The Approach of Acquiring Inspiration: Tacit Understanding and Soul Comprehension}

Different from the "God inspired God-man dual opposition theory" of western aesthetics, Chinese classical aesthetics starts from the conception of philosophy ontology that is the union of nature and man. It always emphasizes the apperception comprehension of the essence of universe and nature. And it acquires inspiration actively from the interaction between the heart and object.

If subconscious information storage is the internal force or internal cause of inspiration, then the prototype enlightenment is the external force or external cause of inspiration. According to the research achievements of modern psychology, inspiration is a kind of cheerful encounter by chance between the subject and object. The forceful stimulation of outside accidental factors forms the brain's nerve center into being intense excitement, which will activate different precipitates in the subconscious mind and combine them to the excitement, to produce high-energy activity effects, to trigger cranial nerve's voltage changes and chemical changes. In this way, inspiration is stimulated. At this moment, the right cerebral hemisphere which is responsible for imaginable thinking will force the left cerebral hemisphere which controls abstract thinking to give way and out of control. This is the condition that soul and object correlated, subject and object unified. Wang Guowei described the condition as: Not knowing what is subject, what is object.

Ancient Chinese starts from this regularity of inspiration to understand the lucky encounter of aesthetic subject and object. In their opinion, the stimulation of inspiration is the process of interaction between the heart and object. This interaction's trapping has two forms: tacit understanding and soul comprehension. The former is direct perception and the latter is intuitive understanding. Their coexistence and complementation constitute the inspiration thinking pattern of 
Chinese classical aesthetics.

First of all, the accidental stimulation of reality is an important motivation of stimulating inspiration. Chinese classical aesthetics considers natural species as the powerful factors that arouse inspiration thinking. When aesthetic subject's psychological pattern accord with nature's inner mechanism to some extent, it is likely to come into being a sudden flash of inspiration, a condition that more affective and creative than ever before. Just as Ge Lifang had said in the Rhythm of poets' note:" comprehending objects with passions, then there will be Xing". At the moment of perceptual tacit understanding, by the artistic intuitive ability, creation subjects can instantly understand the aesthetic connotation that accidental object contains. And they can also dive into the life essence of aesthetic objects. They integrate their emotions into the life of objects, making a fusion of feelings and sceneries; subliming creativity and vitality; delighting emotions and promoting physical and mental pleasure. As the aesthetic experience motivated in instant intuition, the inspiration will be presented in a clear way of thinking. Such a process is just like Yuan Shouding's description in the fifth volume Discussion On The Article of his Zhan Bi Cong Tan, when seeing the view, one will have a coincident feeling and have a wonderful circumstance, as well as the description that preparing everything as need, and waiting a good occasion for emotion in a right way, the action will never out your mind" in Liu Xie's Wen Xin Diao Long.

In this pattern of "tacit understanding", content conception is an important medium opportunity that connects the communication between nature and human and stimulates inspiration. And this content conception is not confined to sceneries. It can be a person, a kind of natural phenomena, an accident, a story, an anecdote and so on. As the accelerant of inspiration, this incidental person, matter and object are frequently fresh and vivid. It is easy to stimulate intuitive comprehension instead of obscure or difficult. At the same time, the information that the stimulant delivers must have a sort of isomorphic correspondence with the aesthetic experience and aesthetic situation that the creation subject accumulates subconsciously. The relationship may complement some element that the new aesthetics presentation group lacks; or it inspires writers to compose new presentation imagination and then to find new aesthetic connotations. Just as Song Lian, a famous literatus in Ming dynasty, had said in Ye Yi Zhong's collected works. when feeling things, there are something in heart, then opening a way for a burst of inspiration. Of course, not all the objective factors can motivate inspiration. This requires creation subjects to have good artistic qualities and intuitive abilities. They are also able to have peculiar sensibility and be observant to surroundings. So that they can seize object's prominent features and aesthetic connotations, and finish the leap from life presentation to art presentation. As Xie Zhen had said that poets need nature's mystery, waiting a good occasion to feel the view and then burst out, although one searches it with all energy, it is not easy to be found. Therefore, Chinese classical aesthetics pays 
more attention to "subtle enlightenment" and subject's spiritual awareness. Usually, it uses some words like "nature's mystery", "God-given inspiration", "sudden enlightenment" to describe inspiration thinking in art creations. For ordinary people, content conception seems common. However, it has profound meaning for these highly sensitive artists. In their eyes, the content conception is the key point of artistic conception and stimulation of inspiration.

Certainly, accidental stimulation is not the only approach to acquire inspiration. Except for relying on objects, subjects can start from memory image and resort to creative imagination to fulfill the creation of art presentation. Chinese classical aestheticians hold the opinion that except for the direct perception: tacit understanding by stimulating, there is another way to acquire inspiration called intuitive perception by initiative acquisition that is through meditation and following natural conscience. Creation subjects overlook the universe in meditation, feeling the natural rhythm of universe and life; experiencing the real existing memory presentation that accumulated in deep-psychology; vivifying the intensions, thoughts, emotions and impressions which potentially live deep in the mind. These elements cohere and integrate with each other to reach a sudden open and clear condition where human and nature united as one, instead of being a chaotic state.

As Xie Zhen's wrote in Si Ming notes on poets, any writing which is written in a quiet room is very hard to created, but never predict that the poet will appear in a sudden. As the wonderful sentences are germinated in heart, the writing brush is also tasted in mouth, no one is left alone to accord with the situation of Xing. And Zhang Yanyuan also wrote in The notes on famous painting of all generations, one should keep the spirit in mind and concentrate his attention to it, as well as Xie Hui, a person in the Ming dynasty, had wrote in The collected works' preface of Fou's sing. keep silent and think to keep spirits to be connected with interests. All these poetic prose demonstrate a kind of contemplation, tacit comprehension and an insight into the slightest details of the universe.

We can see that in the view of Chinese classical aesthetics, there are many approaches of acquiring inspiration, such as touched by the sceneries, intuitive understanding, stimulated by triggering medium, or potential gestation. Of course, both direct perception and intuitive perception are the form of intuitional experience, which don't rely on reasoning. It is the stimulation of information hidden in the brain. And it is a conversion from subconscious to consciousness. Just as the poet Ai Qing said "The acquisition of inspiration is the most cheerful encounter between the poet's subjective and objective world." (Ai, 1979) This encounter is a kind of intuitional experience that looks for movement from stillness. It needs to eliminate other psychological distractions, stimulate neural regions in depressive conditions, and activate some relevant memory presentations that in the deep subconscious mind. Because of this, Chinese classical aestheticians emphasize that the acquisition of inspiration depends on the aesthetic subject to get rid of outside distractions and limitations of sense. They need a 
kind of pure, empty and quiet mentality condition that cannot be disturbed to fully comprehend life consciousness. As Liu Xie's Wen Xin Diao Long Thinking where he said polishing the thinking lies in the visional peace, purifying one's soul and body and washing one's mind in a clean and pure word. In a pure and empty aesthetic mental state, cranial nerve eliminates distractions and works at great tension around some question. All kinds of information materials in the mind are turned over, arranged and processed. As long as the time is ripe for, they would break through the coverage of subconscious, bring consciousness to the surface. Therefore, different from western aesthetics which emphasizes subject's ecstasy; Chinese aesthetics pay more attention to cultivate subject's aesthetic mood state. This empty and quiet state unifies nature and human, purifying the mind and arousing people's interest. Jiao Ran in Tang dynasty had described this kind of state in a poetry that sometimes when encountering a fresh burst of inspiration, there would be a lot of creative creations as if by divine intervention.

\section{The Stimulation and Trapping of Inspiration: "Ingenious Nature without Fabrication" and "Chasing and Catching in a Hurry"}

Though inspiration is very important to the success of creation, it is not an easy or usual option. When talking about aesthetics, Chinese classical aestheticians always emphasize that it is out of human's control. As Yang Wan had wrote in his The three days before midwinter: "one is easy to be drunk, but a piece of poet is got by chance ", the preface of autobiography of Xiao Zixian in the Book of Liang's history had wrote that "Every time, when I want to write something, I am especially lack of the idea of my essay, and it all need to appear by itself instead of using power"; Lu Ji wrote in his Wen Fu: inspiration is based on my mind but its appearance is not controlled by myself. Ancient Chinese find out it is useless to spend time seeking poetry. At the same time, they search effective approaches to acquire inspiration by dialectical thoughts. Based on their creation experience, they made real and graphic revelation of the process of stimulating inspiration.

Chinese aestheticians firstly attach great importance to people's physiological and mental mechanism. They suggest using the brain in a proper and balanced way. Liu Xie give some advice to writer in his Wen Xin Diao Long-thinking, "in writing, please be sure that your heart is clear and bright, and pay more attention on adjusting and dredging to make you have a good temperament. If you are upset, please don't keep on writing, go out and make your mind open. It is that when you have a good mood, you can write something, or you never should write anything. Using a free way to relax yourself, a live and jovial mood to dispel tiredness."

In his opinion, overtime thinking makes the brain overtired and dries up literary thoughts. And if writers violate physiological mechanism to create, at this 
time, it will only disorder the mind. So it is important to adjust and guide human's physiological and mental mechanism in order to clear and gentle the inner heart, then start to create better works. If feeling distracted and thoughts blocked, the creation subject should stop intensive activities and relieve fatigue in a pleased way, so that the inspiration can be stimulated. This is the reason that Xiao Zixian said:"ingenious nature without fabrication."

Now it seems that the classical aesthetics' view of ingenious nature without fabrication accord with the modern psychology's research achievements. Modern psychology thinks that brain's excitement and inhibition adjusted to the best state, is the physiological basis of inspiration production. If the brain has been working for a long time in a highly excited state, it will lead to extreme mental fatigue and inhibit the activity of the cerebral cortex. At this time, if the creation subject gives up focusing on meditation to have a rest. This will distract the subject's attention and inhibit the original excitement center temporarily. But brain cells' activities don't stop completely, and the original surrounding cortex cells turn into excited state. The subconscious mind around the excited center will be stimulated. At that time, the activity of nerve cells increases greatly and a large number of potential stored information is inspired. Then it will get rid of the conventional way of thinking and develop freely and quickly, and break the fixed neural connections under usual inertial thinking mode. In the mean time, various presentations are disrupted and reassembled. The rigid thinking mode is destroyed and then the irregular connections and self-awareness are constructed again. At this moment, once the particularly active subconscious activities are impacted by some factors, they will rise to the ideological level just like the sudden volcanic eruption, and stimulate the burst of inspiration. Xie Lingyun, a famous poet had described his good verse in his dream as "spring grass grows on the side of pond", "the verse is inspired by God". It was obtained under the situation" thinking of poetry all day" while the inspiration appearing when he went to bed.

Therefore, if the writer feels thoughts blocked, there is no need to spend time making some reluctant creations. They might as well try to use the "digress reflection", just as the saying goes" it is a better choice to appreciate flowers and listen to birds singing when there is no creational inspiration." In Guan Zhui Bian, Qian Zhongshu displayed the process and motivating mechanism of inspiration in a vivid and lively way: At the moment of stimulating inspiration, generally the writer has given up long-time contemplation to other things, because further exploration is just a vain attempt except for increasing tension and anxiety. So they'd better start all over again, distract their attentions, change the environment and audio-visual field, and enlarge new information space. During the period of "digress reflection", which seems useless and sell the dummy, though conscious activities have been interrupted, thinking activities don't stop but converting to the level of subconscious mind. A number of information materials enter the subconscious field quickly with the track of former thinking, 
reacted in the information database. An outcome of this is that the ideological gate opens, with sudden enlightenment and flow of inspiration. From this, "digress reflection" is another way to break off the fixed thinking pattern. It seems accidental; in fact, it has other intentions of directionality. The subject's internal feelings and logical laws provide guidance for the writer's thinking. In this way can bring the breakthrough of unusual thinking mode (Qian, 1986).

The burst of inspiration is accidental and fleeting. Su Shi, a great ancient poet, had described the contingency and evanescence of inspiration as, "the moment a hare is flushed out, the falcon swoops down", "subtle verse with fluent expression and quick-thinking", "chase the wind and clutch at shadows". That is to say the inspiration will disappear quickly if not obtained in time. The images in sudden insight are of instability, uncertainty and obscurity. They are partly hidden and partly visible and need the consideration, selection and extraction to become clear and lively. Just as Shao Yong, a philosophe of Song, had said in his Leisurely and comfortable time:"In my spare time, cultivating my spirit by writing make me feel happy, when I have not written anything, I can feel everything around me and forget myself, and the sentences naturally appear in my mind, then the poet is got by chance, there are some mysterious place where I can't grasp, but just a little inspiration will make me be clear and bright. Thus, creation subject needs to properly mobilize and organize all the information materials by consciousness, and make them materialized in text form. By doing this, the clustered images under the depths of subconscious are organized and arranged in order and presented one by one. They may find the main idea, or complete the artistic images, inspire the plot and conception, or stimulate emotions, or collect some good phrases, and so on.

Of course, the control of consciousness should be moderate and cannot be too anxious, otherwise, going too far is as bad as not going far enough. At this point, the inspiration sparks in the bud are fuzzy and fragile. With the collision of consciousness and subconscious, the neuronal connections just established are still fragile. The brain is easily disturbed by distractions, transferring the excitement of cerebral cortex and killing the breakthrough ideas. Maybe it will cut off just established temporary contact between neurons, or cause the ossification and linearization of the contact, or influence each other, causing the disorders of mind and interrupting inspiration. Therefore, it is necessary to capture the inspiration in time and start the creation quickly as soon as inspiration comes. But don't hurry to deliberate and refine details, otherwise, it is easy to block the channel where unconscious activities transfer to consciousness, making inspiration fled in a moment. Of course, the materialization of inspiration requires effective techniques and skills. In the hard process of writing practice, writers not only develop a sensitive thinking and intuition perception, but also become familiar with effective skills of materialization so that they are able to catch and present the sympathetic feelings vividly when inspiration explodes in a moment.

In a word, in the field of Chinese classical aesthetics, inspiration is an unex- 
pected creative sudden insight after the progressive thinking broke off. There exists some inevitable factors behind the contingency: long-time stored knowledge experience provides optional premise for inspiration; long-time research guides the inspiration a right direction to achieve breakthrough; strong emotion requirements and indomitable urge for art objects provide supporting force; abundant energy, acute intuitive perception, accurate strain capacity, and the effective regulation of the brain's excitement and inhibition are the physiological factors; the stimulation of outer information is the external cause of inspiration; a state of relaxed and clear mind is the psychological condition that conceives inspiration; skillful writing skills and literal skills provide necessary materialized foundation for chasing the valuable flash of inspiration timely.

It is worth mentioning that Chinese classical aesthetics' inspiration thinking has the same subtlety with the Buddhism's insight theory: In the way of thinking, it breaks the unexplainable conventionality; on the state of mind, it pays attention to intense meditation and concentration; as for spiritual experience, it is intuitive comprehension that goes beyond utility and correlates soul and object; on the achievements of thinking, it is unique quaintness that known by heart and out of the ordinary; during the process of obtaining mood, it catches unexpected insight after a period of contemplation; as for the external opportunities, it is the unexpected encounter between subjects and objects where the sceneries evoke subject's feelings; about the subjective conditions, it is an unexpected cherishing by chance after voluminous knowledge accumulation and treasure storage.

Chinese classical aestheticians start from their own creation experience, on the base of the simple theory of interaction between the heart and the object, they make a real, tactful explanation and revelation to the thinking characters, artistic functions, acquiring methods, mental mechanism, gestation and catching of inspiration. This revelation doesn't attach importance to the structure of logic, critical thought and system. It pays more attention to the life experience, intuitional understanding and description of inspiration. It values the creator's life experience and knowledge accumulation and subject's spiritual awareness. And it attaches importance to the cultivation of the subject's inherent spirit, creation sentiment and aesthetic mental state. It values spiritual accordance and interaction between aesthetic subjects and objects. And it also encourages acquiring inspiration actively from the interaction between the heart and object for the moment. Though this theory of inspiration is not the same incisive as the western aesthetics, it has its own flexible explanation space and it reflects Chinese classical aestheticians' precise cognition to the psychological characteristics of art creation. It has commensurability with western inspiration theory. Both can be implemented without coming into conflict. As a result, taking the theory of modern aesthetics and psychology as reference, digging deep into the Chinese classical aesthetics' radiance of theory, and finding out the common principle of aesthetics between Chinese literature and western art, is undoubtedly an important academic sector that is worth researching and concerning under the context 
of global culture.

\section{Conclusion}

Chinese traditional aesthetics of inspiration thinking can be roughly divided into two kinds: the type of intuitive perception triggered inspiration and the type of intuition understanding spontaneous inspiration. Both are intuitive experience, not involved in reasoning, paying great attention to the "wonderful" and the writer's spiritual thinking. Chinese style inspiration thinking and Buddhist enlightenment have many similarities: in the way of thinking, they are innovative thinking, break through the thinking set, can only understand but cannot be expressed in words; in the creation of the state of mind, they are empty static state, without distraction; in the spiritual experience, they are intuitive wisdom, beyond utilitarian, all-inclusive, heart and things together; in the thinking achievements, they are unique, extraordinary and full of wit and humour; in the process, they have experienced a cudgel thinking and suddenly see the light; in the external opportunities, they are moved by sight, accidentally across between the subject and object; in terms of the subject, they all require long-term creative training and get excellent works by chance with a high skill.

\section{References}

Ai, Q. (1979). The Poet Speaks The Truth. Nan Fang Daily, 2.

Qian, Z. S. (1984). Notes on Literature and Art. Zhong Hua Book, 272-275.

Qian Z. S. (1986). Guan Zhui Bian. Zhong Hua Book, 1186.

Zheng, Q. Y., \& Li, X. D. (1987). The History of Chinese Aesthetics. Hebei Peoples Publishing House, 139-142. 